\title{
Multi-objective Assignment Problem with Fuzzy Costs for the Case of Military Affairs
}

\author{
Pranab Biswas \\ Assistant Teacher \\ Sardanga High School \\ P.O.- Chakdaha, Nadia, \\ West Bengal, 741222, India
}

\author{
Surapati Pramanik \\ Assistant Professor \\ Nandalal Ghosh B.T. College, Panpur, \\ P.O.- Narayanpur, North 24 Parganas \\ West Bengal, 743126, India
}

\begin{abstract}
This paper presents multi-objective assignment problem with fuzzy costs, where all the objectives are to be simultaneously minimized. Here each fuzzy cost is assumed as trapezoidal fuzzy number. To form a single objective problem of a multiobjective fuzzy assignment problem, weights of the objectives have been taken according to their priorities. Yager's ranking method has been used to transform a newly formed single objective fuzzy assignment problem into a crisp assignment problem in the linear programming problem form. Then, the single objective linear programming can be solved by any conventional method. A numerical example is provided to demonstrate the potentiality of the proposed approach.
\end{abstract}

\section{General terms}

Fuzzy multi-objective assignment problem.

\section{Key words}

Multi-objective assignment problem, Trapezoidal fuzzy number, Yager's ranking method, Fuzzy set, Fuzzy number.

\section{INTRODUCTION}

An assignment problem (AP) is a particular type of transportation problem where $\mathrm{n}$ tasks (jobs) are to be assigned to an equal number of $n$ machines (workers) in one to one basis such that the assignment cost (or profit) is minimum (or maximum). Hence, it can be considered as a balanced transportation problem in which all supplies and demands are equal, and the number of rows and columns in the matrix are identical. Different type of assignment problems for a single objective function in crisp environment is discussed in the literature [1-4]. Bao et al. [5] studied the multi-objective assignment problem (MOAP) in crisp environment. Lin and Wen [6] proposed labelling algorithm to solve assignment problem with fuzzy interval cost. Chen [7] proposed fuzzy assignment model by considering that all the individuals involved have the same skills. Wang [8] solved a similar model by graph theory. Mukherjee and Basu [9] solved an assignment problem with fuzzy cost by Yager's ranking method [10] that transforms the fuzzy assignment problem into a crisp assignment problem. For a multi-objective assignment problem, Geetha et al. [11] expressed an assignment problem that minimizes both time and cost. Tsai et al.[12] discussed a multi-objective decision making problem associated with cost time or quality by fuzzy parameters. They extended the traditional single objective assignment problem to the multi objective decision making problem associated with cost, time and quality. Kagade and Bajaj [13] discussed MOAP where the cost coefficients of the objective functions are interval numbers. Kagade and Bajaj [14] also solved a MOAP by using some linear and non linear membership functions. De and Yadav [15] discussed fuzzy multi-objective assignment problem (FMOAP) through interactive fuzzy goal programming approach. Pramanik et al. [16] discussed the fuzzy goal programming approach for multiobjective transportation, with crisp and fuzzy coefficients. Pramanik et al. [17] studied the priority based goal programming approach for multi-objective transportation problem with fuzzy parameters.

In this paper, we consider MOAP with fuzzy parameters for the case of military affairs. Let $\left(\tilde{\mathrm{C}}_{\mathrm{ij}}\right)$ be assigned cost for fighter plane-i to perform the $\mathrm{j}$-th task. Assume that $\tilde{\mathrm{t}}_{\mathrm{ij}}$ denotes the time used to complete the $j$-th task by fighter plane-i and $\tilde{q}_{i j}$ denotes the inefficiency of the fighter plane-i to perform the j-th task. Let us assume that operation cost, operation time and inefficiency are trapezoidal fuzzy numbers. However, due to different unit of cost, time, ineffectiveness, it is not possible to merge each other until we normalize them. For normalization purpose, we divide operation cost, time, and ineffectiveness by their corresponding maximum ranking index, determined by Yager's ranking method. To obtain a resultant single objective function, weights have been assigned to the objectives according to their priorities. Now by Yager's ranking method, we can transform a newly formed single objective fuzzy assignment problem to a crisp assignment problem in linear programming problem form. Then it can be solved by any conventional method.

The rest of the paper is organized as follows. In section 2, preliminaries of fuzzy sets are presented. In section 3 , we discuss multi-objective assignment problem. In section 4 , the proposed model on fuzzy multi-objective assignment problem has been given. An example has been given to demonstrate the effectiveness of the proposed method in section 5. Finally, section 6 presents the conclusions. 


\section{PRELIMINARIES OF FUZZY SETS 2.1 Definition}

Fuzzy set: A fuzzy set $\tilde{\mathrm{A}}$ in a universe of discourse $X$ is defined by $\tilde{\mathrm{A}}=\left\{\left\langle\mathrm{x}, \mu_{\tilde{\mathrm{A}}}(\mathrm{x})\right\rangle \mid \mathrm{x} \in X\right\}$, where $\mu_{\tilde{\mathrm{A}}}(\mathrm{x}): X \rightarrow[0,1]$ is called the membership function of $\tilde{\mathrm{A}}$ and $\mu_{\tilde{\mathrm{A}}}(\mathrm{x})$ is the degree of membership to which $\mathrm{x} \in \tilde{\mathrm{A}}$.

\subsection{Definition}

A fuzzy set $\tilde{\mathrm{A}}$ on $R$ is convex if and only if for any $\mathrm{x}_{1}, \mathrm{x}_{2} \in X$, the membership function of $\tilde{\mathrm{A}}$ satisfies the inequality $\mu_{\tilde{\mathrm{A}}}\left(\lambda \mathrm{x}_{1}+(1-\lambda) \mathrm{x}_{2}\right) \geq \min \left\{\mu_{\tilde{\mathrm{A}}}\left(\mathrm{x}_{1}\right), \mu_{\tilde{\mathrm{A}}}\left(\mathrm{x}_{2}\right)\right\} ; 0 \leq \lambda \leq 1$.

\subsection{Definition}

A fuzzy set $\tilde{\mathrm{A}}$ of the universe of discourse $X$ is called a normal fuzzy set if there exists at least one $\mathrm{x} \in X$ such that $\mu_{\tilde{\mathrm{A}}}(\mathrm{x})=1$.

\subsection{Definition}

A fuzzy set $\tilde{\mathrm{A}}$, defined on the universal set of real number $R$, is said to be a fuzzy number, if its membership function has the following characteristics. i) $\tilde{\mathrm{A}}$ is convex, ii) $\tilde{\mathrm{A}}$ is normal and iii) $\mu_{\tilde{\mathrm{A}}}(\mathrm{x})$ is piecewise continuous.

\subsection{Definition}

A trapezoidal fuzzy number $\tilde{a}$ is denoted by $\left(a_{1}, a_{2}, a_{3}, a_{4}\right)$ where $a_{1}, a_{2}, a_{3}, a_{4}$ are real numbers and its membership function $\mu_{\tilde{a}}(\mathrm{x})$ is given by

$$
\mu_{\tilde{a}}(x)=\left\{\begin{array}{ll}
0, & x \leq a_{1}, \\
\frac{x-a_{1}}{a_{2}-a_{1}}, & a_{2} \leq x \leq a_{1}, \\
1, & a_{2} \leq x \leq a_{3}, \\
\frac{a_{4}-x}{a_{4}-a_{3}}, & a_{3} \leq x \leq a_{4}, \\
0, & x \geq a_{4}
\end{array}\right\}
$$

$\mu_{\tilde{\mathrm{a}}}(\mathrm{x})$ satisfies the following conditions.

1. $\mu_{\tilde{\mathrm{a}}}(\mathrm{x})$ is a continuous mapping from $R$ to closed interval $[0,1]$

2. $\mu_{\tilde{a}}(x)=0$ for every $x \in\left(-\infty, a_{1}\right]$

3. $\mu_{\tilde{a}}(x)$ is strictly increasing and continuous on $\left[a_{1}, a_{2}\right]$

4. $\mu_{\tilde{a}}(x)=1$ for every $x \in\left[a_{2}, a_{3}\right]$

5. $\mu_{\tilde{\mathrm{a}}}(\mathrm{x})$ is strictly decreasing and continuous on $\left[\mathrm{a}_{3}, \mathrm{a}_{4}\right]$

6. $\mu_{\tilde{a}}(\mathrm{x})=0$ for every $\mathrm{x} \in\left[\mathrm{a}_{4}, \infty\right)$

\subsubsection{Definition}

The $\alpha$-cutset of a fuzzy set $\tilde{\mathrm{A}}$ is a crisp set defined by $\tilde{\mathrm{A}}_{\alpha}=\left\{\mathrm{x} \in \mathrm{X} / \mu_{\tilde{\mathrm{A}}}(\mathrm{x}) \geq \alpha\right\}$.

\subsection{Definition}

For a convex fuzzy number $\tilde{\mathrm{C}}$, the Yager's Ranking index

$\mathrm{Y}(\tilde{\mathrm{C}})$ is defined by $\mathrm{Y}(\tilde{\mathrm{C}})=\int_{0}^{1} 0.5\left(\mathrm{C}_{\alpha}^{\mathrm{L}}+\mathrm{C}_{\alpha}^{\mathrm{U}}\right) \mathrm{d} \alpha$

Here $\left(C_{\alpha}^{L}, C_{\alpha}^{U}\right)$ is a $\alpha$-level cut of fuzzy number $\tilde{C}$.
The index gives the representative value of fuzzy number $(\tilde{\mathrm{C}})$ If $\tilde{\mathrm{E}}=\mathrm{a} \tilde{\mathrm{F}}+\mathrm{b} \tilde{\mathrm{G}}$ and $\tilde{\mathrm{H}}=\mathrm{c} \tilde{\mathrm{I}}-\mathrm{d} \tilde{\mathrm{J}}$, where $\mathrm{a}, \mathrm{b}, \mathrm{c}$ and $\mathrm{d}$ are constants. Then $\mathrm{Y}(\tilde{\mathrm{E}})=\mathrm{aY}(\tilde{\mathrm{F}})+\mathrm{bY}(\tilde{\mathrm{G}})$ and $\mathrm{Y}(\tilde{\mathrm{H}})=\mathrm{cY}(\tilde{\mathrm{I}})-\mathrm{dY}(\tilde{\mathrm{J}})$ can be written based on linear and additive properties of Yager's ranking method.

\section{MODEL CONSTRUCTION OF MULTI- OBJECTIVE ASSIGNMENT PROBLEM (MOAP)}

In a simple $\mathrm{AP}, \mathrm{n}$ jobs are to be performed by $\mathrm{n}$ persons depending on their efficiency to do the job in one to one basis such that the assignment cost is minimal. Now if the objective of an AP is to minimize operation cost, operation time, and poor quality (quantified as a real number), then we treat this type of problem as a multi objective AP. Here, we consider assignment problem with three objectives in the following form of $n \times n$ cost matrix where each cell having a cost $\left(c_{i j}\right)$, time $\left(t_{i j}\right)$, quality $\left(\mathrm{q}_{\mathrm{ij}}\right)$, all of them are real numbers as shown in Table 1 .

Table 1. Presentation of cost $(C)$, time (T) and quality $(Q)$ of fighter plane (FP)

\begin{tabular}{|c|c|c|c|}
\hline \multicolumn{1}{|c|}{ Q FP, $\mathrm{T}}$, & Task-1 & $\ldots$ Task-j $\ldots .$. & Task-n \\
\hline FP-1 & $\mathrm{c}_{11} ; \mathrm{t}_{11} ; \mathrm{q}_{11}$ & $\ldots \mathrm{c}_{1 \mathrm{j}} ; \mathrm{t}_{1 \mathrm{j}} ; \mathrm{q}_{1 \mathrm{j}} \ldots$ & $\mathrm{c}_{1 \mathrm{n}} ; \mathrm{t}_{1 \mathrm{n}} ; \mathrm{q}_{1 \mathrm{n}}$ \\
\hline$\ldots$ & $\ldots$ & $\ldots$ & $\ldots$ \\
\hline FP-i & $\mathrm{c}_{\mathrm{i} 1} ; \mathrm{t}_{\mathrm{i} 1} ; \mathrm{q}_{\mathrm{i} 1}$ & $\ldots \mathrm{c}_{\mathrm{ij}} ; \mathrm{t}_{\mathrm{ij}} ; \mathrm{q}_{\mathrm{ij}} \ldots$ & $\mathrm{c}_{\mathrm{in}} ; \mathrm{t}_{\mathrm{in}} ; \mathrm{q}_{\mathrm{in}}$ \\
\hline$\ldots$ & $\ldots$ & $\ldots$ & $\ldots$ \\
\hline FP-n & $\mathrm{c}_{\mathrm{n} 1} ; \mathrm{t}_{\mathrm{n} 1} ; \mathrm{q}_{\mathrm{n} 1}$ & $\ldots \mathrm{c}_{\mathrm{ni}} ; \mathrm{t}_{\mathrm{ni}} ; \mathrm{q}_{\mathrm{ni}} \ldots$ & $\mathrm{c}_{\mathrm{nn}} ; \mathrm{t}_{\mathrm{nn}} ; \mathrm{q}_{\mathrm{nn}}$ \\
\hline
\end{tabular}

Since each task is assigned strictly one to one fighter plane. Mathematically, the equivalent single objective AP of the corresponding MOAP can be stated due to Bao et al. [5] as follows.

$\operatorname{Min}(\mathrm{C}, \mathrm{T}, \ldots ., \mathrm{Q})=$

$w_{1} \sum_{i=1}^{n} \sum_{j=1}^{n} c_{i j} x_{i j}+w_{2} \sum_{i=1}^{n} \sum_{j=1}^{n} t_{i j} x_{i j}+\ldots \ldots \ldots \ldots . . . . .+w_{n} \sum_{i=1}^{n} \sum_{j=1}^{n} q_{i j} x_{i j}$

$\sum_{\mathrm{i}=1}^{\mathrm{n}} \mathrm{x}_{\mathrm{ij}}=1 ; \sum_{\mathrm{j}=1}^{\mathrm{n}} \mathrm{x}_{\mathrm{ij}}=1$; and $\sum \mathrm{w}_{\mathrm{i}}=1$

$\mathrm{x}_{\mathrm{ij}}=1$, task $\mathrm{j}$ is assigned to $\mathrm{FP}-\mathrm{i}$

$=0$, otherwise

In equation (2) $\mathrm{c}_{\mathrm{ij}}, \mathrm{t}_{\mathrm{ij}}$, and $\mathrm{q}_{\mathrm{ij}}$ are represented as normalized cost, time and quality respectively. $\mathrm{w}_{1}, \mathrm{w}_{2}, \ldots, \mathrm{w}_{\mathrm{n}}$ are the corresponding weights of objectives, (cost, time and quality) according their importance. Normalization of operation cost, 
time, and quality has been done, dividing each of them by their individual maximum numerical value say, $1 / \mathrm{k}_{1}$ for cost, $1 / \mathrm{k}_{2}$ for time, $1 / \mathrm{k}_{3}$ for quality or ineffectiveness. Therefore to perform the Task- $j$, for FP-i we have normalized cost as $k_{1} c_{i j}$, normalized time as $\mathrm{k}_{2} \mathrm{t}_{\mathrm{ij}}$ normalized ineffectiveness as $\mathrm{k}_{3} \mathrm{q}_{\mathrm{ij}}$, $j=1,2, \ldots, n, i=1,2, \ldots, n$. The normalized data will not affect the assignment problem.

In realistic sense, cost, time and quality of MOAP cannot be taken as a crisp value every time. Then, costs, time and quality of MOAP are characterized by uncertain information such as fuzzy variables. We consider here, the cost $\left(\tilde{c}_{i j}\right)$, time $\left(\tilde{t}_{i j}\right)$ and quality $\left(\tilde{\mathrm{q}}_{\mathrm{ij}}\right)$ of fuzzy variables are represented as trapezoidal fuzzy numbers. As a result, each objective becomes fuzzy number and it cannot be minimized directly. For such a situation, we first determine the Yager's ranking index of each fuzzy cost, fuzzy time, and fuzzy ineffectiveness by Yager's ranking method. From these values, we get the maximum ranking index of fuzzy cost, fuzzy time, and fuzzy ineffectiveness respectively. With the help of corresponding maximum value, normalized value can be obtained.

\section{FUZZY MULTI-OBJECTIVE ASSIGNMENT PROBLEM (FMOAP)}

Mathematical model of multi-objective fuzzy assignment problem can be stated as follows:

Minimize $\mathrm{Z}_{\mathrm{k}}(\overline{\mathrm{X}})=\sum_{\mathrm{i}=1 \mathrm{j}=1}^{\mathrm{n}} \sum_{\mathrm{ij}}^{\mathrm{n}} \tilde{\mathrm{C}}_{\mathrm{ij}}^{\mathrm{k}} \mathrm{X}_{\mathrm{ij}}, \mathrm{k}=1,2, \ldots, \mathrm{n}$

subject to $Z_{k}(X)=\left\{Z_{1}(X), Z_{2}(X), \ldots, Z_{k}(X)\right\}$

$\sum_{i=1}^{n} X_{i j}=1, j=1,2, \ldots, n$. and $\sum_{j=1}^{n} X_{i j}=1, i=1,2, . ., n$.

where $Z_{k}(X)=\left\{Z_{1}(X), Z_{2}(X), \ldots, Z_{k}(X)\right\}$ is a vector of $K$ objective functions. If $\mathrm{Z}_{1}(\mathrm{X})$ denotes cost function, $\mathrm{Z}_{2}(\mathrm{X})$ denotes time function, and $Z_{3}(X)$ denotes the quality function, described as a fuzzy number, then it is a three objective fuzzy assignment problem

To construct a single-objective problem of a three objective fuzzy assignment problem, we first normalize the fuzzy cost $\left(\tilde{\mathrm{c}}_{\mathrm{ij}}\right)$, fuzzy time $\left(\tilde{\mathrm{t}}_{\mathrm{ij}}\right)$ and ineffectiveness $\left(\tilde{\mathrm{q}}_{\mathrm{ij}}\right)$ and use weight to consider the priorities of the objective.

$\operatorname{Min}(\tilde{\mathrm{C}}, \tilde{\mathrm{T}}, \tilde{\mathrm{Q}})=$

$\mathrm{w}_{1} \sum_{\mathrm{i}=1}^{\mathrm{n}} \sum_{\mathrm{j}=1}^{\mathrm{n}}\left(\tilde{\mathrm{c}}_{\mathrm{ij}}\right) \mathrm{x}_{\mathrm{ij}}+\mathrm{w}_{2} \sum_{\mathrm{i}=1}^{\mathrm{n}} \sum_{\mathrm{j}=1}^{\mathrm{n}}\left(\tilde{\mathrm{t}}_{\mathrm{ij}}\right) \mathrm{x}_{\mathrm{ij}}+\mathrm{w}_{3} \sum_{\mathrm{i}=1}^{\mathrm{n}} \sum_{\mathrm{j}=1}^{\mathrm{n}}\left(\tilde{\mathrm{q}}_{\mathrm{ij}}\right) \mathrm{x}_{\mathrm{ij}}$

subject to $\sum_{\mathrm{i}=1}^{\mathrm{n}} \mathrm{x}_{\mathrm{ij}}=1 ; \sum_{\mathrm{j}=1}^{\mathrm{n}} \mathrm{x}_{\mathrm{ij}}=1$. where $\mathrm{w}_{1}+\mathrm{w}_{2}+\mathrm{w}_{3}=1$.

Since Yager's Ranking Method satisfying the linearity and additive properties, then,
$\operatorname{Min} Y(\tilde{\mathrm{C}}, \tilde{\mathrm{T}}, \tilde{\mathrm{Q}})=$

$w_{1} \sum_{i=1}^{n} \sum_{j=1}^{n} Y\left(\tilde{c}_{i j}\right) x_{i j}+w_{2} \sum_{i=1}^{n} \sum_{j=1}^{n} Y\left(\tilde{t}_{i j}\right) x_{i j}+w_{3} \sum_{i=1}^{n} \sum_{j=1}^{n} Y\left(\tilde{q}_{i j}\right) x_{i j}$

s.t. $\quad \sum_{i=1}^{n} x_{i j}=1 ; \sum_{j=1}^{n} x_{i j}=1$. Where $w_{1}+w_{2}+w_{3}=1$.

$\mathrm{x}_{\mathrm{ij}}=1$, task $\mathrm{j}$ is assigned to FP- $\mathrm{i}$.

$=0$, otherwise.

Where $\mathrm{x}_{\mathrm{ij}}$ is the decision variable denoting the assignment of the FP- $i$ to task $j$ and cost $\left(\tilde{c}_{i j}\right)$, time $\left(\tilde{t}_{i j}\right)$ and quality $\left(\tilde{\mathrm{q}}_{\mathrm{ij}}\right)$ have their own meanings (See Table 2).

Table 2. Fuzzy cost, fuzzy time and ineffectiveness data of fuzzy assignment problem

\begin{tabular}{|c|c|c|c|}
\hline \multicolumn{1}{c|}{ C, T, Q } & Task-1 & $\ldots$ Task-j $\ldots$ & Task-n \\
\hline FP-1 & $\tilde{c}_{11} ; \tilde{\mathrm{t}}_{11} ; \tilde{\mathrm{q}}_{11}$ & $\ldots \tilde{\mathrm{c}}_{1 \mathrm{j}} ; \tilde{\mathrm{t}}_{1 \mathrm{j}} ; \tilde{\mathrm{q}}_{1 \mathrm{j}} \ldots$ & $\tilde{\mathrm{c}}_{\mathrm{ln}} ; \tilde{\mathrm{t}}_{\mathrm{tn}} ; \tilde{\mathrm{q}}_{1 \mathrm{n}}$ \\
\hline$\ldots$ & $\ldots$ & $\ldots$ & $\ldots$ \\
\hline FP-i & $\tilde{\mathrm{c}}_{\mathrm{i} 1} ; \tilde{\mathrm{t}}_{\mathrm{i} 1} ; \tilde{\mathrm{q}}_{\mathrm{i} 1}$ & $\ldots \tilde{\mathrm{c}}_{\mathrm{ij}} ; \tilde{\mathrm{t}}_{\mathrm{ij}} ; \tilde{\mathrm{q}}_{\mathrm{ij}} \ldots$ & $\tilde{\mathrm{c}}_{\mathrm{in}} ; \tilde{\mathrm{t}}_{\mathrm{in}} ; \tilde{\mathrm{q}}_{\mathrm{in}}$ \\
\hline$\ldots$. & $\ldots$. & $\ldots$ & $\ldots$ \\
\hline FP-n & $\tilde{\mathrm{c}}_{\mathrm{n} 1} ; \tilde{\mathrm{t}}_{\mathrm{n} 1} ; \tilde{\mathrm{q}}_{\mathrm{n} 1} \ldots \tilde{\mathrm{c}}_{\mathrm{nj}} ; \tilde{\mathrm{t}}_{\mathrm{nj}} ; \tilde{\mathrm{q}}_{\mathrm{nj}} \ldots$ & $\tilde{\mathrm{c}}_{\mathrm{nn}} ; \tilde{\mathrm{t}}_{\mathrm{nn}} ; \tilde{\mathrm{q}}_{\mathrm{nn}}$ \\
\hline
\end{tabular}

\section{NUMERICAL EXAMPLES}

Let us consider assignment problem with three objectives for the case of military affairs. Information about operation cost, operation time and quality of fighter planes (FPs) have been given in Table 3 according to their suitable units.

Solution: To solve the problem, we have to determine the Yager's ranking index of cost, time and ineffectiveness represented here as trapezoidal fuzzy numbers. We calculate $Y(4,6,7,9)$ by applying Yager's Ranking method . The $\alpha$-cut of fuzzy number $(4,6,7,9)$ is $\left(\mathrm{C}^{\mathrm{L}} \alpha, \mathrm{C}^{\mathrm{U}}{ }_{\alpha}\right)=(2 \alpha+4,9-2 \alpha)$ for which

$\mathrm{Y}\left(\tilde{\mathrm{C}}_{11}\right)=\mathrm{Y}(4,6,7,9)=\int_{0}^{1} 0.5\left(\mathrm{C}^{\mathrm{L}} \alpha+\mathrm{C}^{\mathrm{U}} \alpha\right) \mathrm{d} \alpha=6.5$

$\mathrm{Y}(7,9,11,13)=10 ; \mathrm{Y}(0.15,0.16,0.19,0.21)=0.1775 ;$

Similarly, we get the other Yager ranking index with respect to their corresponding element. $\mathrm{Y}\left(\tilde{\mathrm{c}}_{12}\right)=6.25 ; \mathrm{Y}\left(\tilde{\mathrm{t}}_{12}\right)=9.25$;

$\mathrm{Y}\left(\tilde{\mathrm{q}}_{12}\right)=0.12 . \mathrm{Y}\left(\tilde{\mathrm{c}}_{13}\right)=8.75 ; \mathrm{Y}\left(\tilde{\mathrm{t}}_{13}\right)=10.5 ; \mathrm{Y}\left(\tilde{\mathrm{q}}_{13}\right)=0.17$.

$\mathrm{Y}\left(\tilde{\mathrm{c}}_{14}\right)=5.5 ; \quad \mathrm{Y}\left(\tilde{\mathrm{t}}_{14}\right)=\mathbf{1 1 . 7 5} ; \mathrm{Y}\left(\tilde{\mathrm{q}}_{14}\right)=0.08 . \quad \mathrm{Y}\left(\tilde{\mathrm{c}}_{21}\right)=4.25 ;$

$\mathrm{Y}\left(\tilde{\mathrm{t}}_{21}\right)=8.75 ; \quad \mathrm{Y}\left(\tilde{\mathrm{q}}_{21}\right)=0.135 . \mathrm{Y}\left(\tilde{\mathrm{c}}_{22}\right)=6.25 ; \quad \mathrm{Y}\left(\tilde{\mathrm{t}}_{22}\right)=10 ;$ 
$\mathrm{Y}\left(\tilde{\mathrm{q}}_{22}\right)=0.17 . \mathrm{Y}\left(\tilde{\mathrm{c}}_{23}\right)=7 ; \mathrm{Y}\left(\tilde{\mathrm{t}}_{23}\right)=9 ; \mathrm{Y}\left(\tilde{\mathrm{q}}_{23}\right)=0.2225 ;$

$\mathrm{Y}\left(\tilde{\mathrm{c}}_{24}\right)=7.75 ; \mathrm{Y}\left(\tilde{\mathrm{t}}_{24}\right)=9.75 ; \mathrm{Y}\left(\tilde{\mathrm{q}}_{24}\right)=0.20 ; \mathrm{Y}\left(\tilde{\mathrm{c}}_{31}\right)=9 ; \mathrm{Y}($

$\left.\tilde{\mathrm{t}}_{31}\right)=4.75 ; \mathrm{Y}\left(\tilde{\mathrm{q}}_{31}\right)=0.21 ; \mathrm{Y}\left(\tilde{\mathrm{c}}_{32}\right)=9.5 ; \mathrm{Y}\left(\tilde{\mathrm{t}}_{32}\right)=6.25 ; \mathrm{Y}($

$\left.\tilde{\mathrm{q}}_{32}\right)=0.16 ; \mathrm{Y}\left(\tilde{\mathrm{c}}_{33}\right)=8 ; \mathrm{Y}\left(\tilde{\mathrm{t}}_{33}\right)=8 ; \mathbf{Y}\left(\tilde{\mathrm{q}}_{33}\right)=\mathbf{0 . 2 3 2 5} ; \quad \mathrm{Y}($

$\left.\tilde{\mathrm{c}}_{34}\right)=6.25 ; \mathrm{Y}\left(\tilde{\mathrm{t}}_{34}\right)=5 ; \mathrm{Y}\left(\tilde{\mathrm{q}}_{34}\right)=0.1725 ; \mathrm{Y}\left(\tilde{\mathrm{c}}_{41}\right)=8 ; \quad \mathrm{Y}($

$\left.\tilde{\mathrm{t}}_{41}\right)=7 ; \mathrm{Y}\left(\tilde{\mathrm{q}}_{41}\right)=0.1875 ; \mathrm{Y}\left(\tilde{\mathrm{c}}_{42}\right)=8.75 ; \mathrm{Y}\left(\tilde{\mathrm{t}}_{42}\right)=7.5 ; \mathrm{Y}($

$\left.\tilde{\mathrm{q}}_{42}\right)=0.22 ; \mathrm{Y}\left(\tilde{\mathrm{c}}_{43}\right)=\mathbf{1 0 . 7 5} ; \mathrm{Y}\left(\tilde{\mathrm{t}}_{43}\right)=6 ; \mathrm{Y}\left(\tilde{\mathrm{q}}_{43}\right)=0.135 ; \mathrm{Y}($

$\left.\tilde{\mathrm{c}}_{44}\right)=9 ; \mathrm{Y}\left(\tilde{\mathrm{t}}_{44}\right)=10.75 ; \mathrm{Y}\left(\tilde{\mathrm{q}}_{44}\right)=0.145$

So maximum cost of this fuzzy cost matrix is $\mathrm{Y}\left(\tilde{\mathrm{C}}_{43}\right)=10.75$; maximum time $\mathrm{Y}\left(\tilde{\mathrm{t}}_{14}\right)=11.75$ and maximum of ineffectiveness is $\mathrm{Y}\left(\tilde{\mathrm{C}}_{33}\right)=0.2325$. We obtained normalized operation cost, operation time, and ineffectiveness (See Table-4).

We have $\operatorname{Max}\left\{Y_{c}\right\}=10.75=1 / k_{1}$ (say), similarly, $\operatorname{Max}\left\{Y_{t}\right\}=1 / k_{2}$ $\operatorname{Max}\left\{Y_{\text {in }}\right\}=1 / k_{3}$ where $Y_{c}, Y_{t}, Y_{\text {in }}$ indicate the Yager's ranking of cost, time, and ineffective respectively. The objective of this assignment problem is to look for minimized cost, time and ineffectiveness. We assume the weights for each objective as $\mathrm{w}_{1}$, $w_{2}, w_{3}$. Let us assume that $a=w_{1} k_{1}, b=w_{2} k_{2}$ and $c=w_{3} k_{3}$. Then, the FMOAP can be formulated in the following mathematical programming form:

$\operatorname{Min}\left[\sum_{\mathrm{i}=1 \mathrm{j}=1}^{\mathrm{n}} \sum^{\mathrm{n}} \mathrm{Y}\left(\mathrm{a} \tilde{\mathrm{c}}_{\mathrm{ij}}+\mathrm{b} \tilde{\mathrm{t}}_{\mathrm{ij}}+\mathrm{c} \tilde{\mathrm{q}}_{\mathrm{ij}}\right) \mathrm{x}_{\mathrm{ij}}\right]$

subject to

$\mathrm{x}_{11}+\mathrm{x}_{12}+\mathrm{x}_{13}+\mathrm{x}_{14}=1, \mathrm{x}_{11}+\mathrm{x}_{21}+\mathrm{x}_{31}+\mathrm{x}_{41}=1$,

$\mathrm{x}_{21}+\mathrm{x}_{22}+\mathrm{x}_{23}+\mathrm{x}_{24}=1, \mathrm{x}_{12}+\mathrm{x}_{22}+\mathrm{x}_{32}+\mathrm{x}_{42}=1$,

$\mathrm{x}_{31}+\mathrm{x}_{32}+\mathrm{x}_{33}+\mathrm{x}_{34}=1, \mathrm{x}_{13}+\mathrm{x}_{22}+\mathrm{x}_{33}+\mathrm{x}_{43}=1$,

$\mathrm{x}_{41}+\mathrm{x}_{42}+\mathrm{x}_{43}+\mathrm{x}_{44}=1, \mathrm{x}_{14}+\mathrm{x}_{24}+\mathrm{x}_{34}+\mathrm{x}_{44}=1$, where $\mathrm{x}_{\mathrm{ij}} \in\{0,1\}$.

Since the Yager's Ranking method satisfies the linearity and additive property, the problem can be rewritten as follows:

$\operatorname{Min} \sum_{\mathrm{i}=1 \mathrm{j}=\mathrm{i}}^{4}\left\{\mathrm{aY}\left(\tilde{\mathrm{c}}_{\mathrm{ij}}\right)+\mathrm{bY}\left(\tilde{\mathrm{t}}_{\mathrm{ij}}\right)+\mathrm{cY}\left(\tilde{\mathrm{q}}_{\mathrm{ij}}\right)\right\} \mathrm{x}_{\mathrm{ij}}$

subject to

$\mathrm{x}_{11}+\mathrm{x}_{12}+\mathrm{x}_{13}+\mathrm{x}_{14}=1, \mathrm{x}_{11}+\mathrm{x}_{21}+\mathrm{x}_{31}+\mathrm{x}_{41}=1$,

$\mathrm{x}_{21}+\mathrm{x}_{22}+\mathrm{x}_{23}+\mathrm{x}_{24}=1, \mathrm{x}_{12}+\mathrm{x}_{22}+\mathrm{x}_{32}+\mathrm{x}_{42}=1$,

$\mathrm{x}_{31}+\mathrm{x}_{32}+\mathrm{x}_{33}+\mathrm{x}_{34}=1, \mathrm{x}_{13}+\mathrm{x}_{22}+\mathrm{x}_{33}+\mathrm{x}_{43}=1$,

$\mathrm{x}_{41}+\mathrm{x}_{42}+\mathrm{x}_{43}+\mathrm{x}_{44}=1, \mathrm{x}_{14}+\mathrm{x}_{24}+\mathrm{x}_{34}+\mathrm{x}_{44}=1$,

where $\mathrm{x}_{\mathrm{ij}} \in\{0,1\}$.

Setting the value of $\mathrm{w}_{1}=0.2, \mathrm{w}_{2}=0.3, \mathrm{w}_{3}=0.5, \mathrm{k}_{1}=0.0930$, $\mathrm{k}_{2}=0.0851$, and $\mathrm{k}_{3}=0.0432$, we have

Min $\left[0.7569 \mathrm{x}_{11}+0.6105 \mathrm{x}_{12}+0.7964 \mathrm{x}_{13}+0.5743 \mathrm{x}_{14}+0.5928\right.$

$\mathrm{x}_{21}+0.7372 \mathrm{x}_{22}+0.8385 \mathrm{x}_{23}+0.8232 \mathrm{x}_{24}+0.7403 \mathrm{x}_{31}+$

$0.6804 \mathrm{x}_{32}+0.8531 \mathrm{x}_{33}+0.6149 \mathrm{x}_{34}+0.7308 \mathrm{x}_{41}+0.8274 \mathrm{x}_{42}$

$+0.6435 \mathrm{x}_{43}+0.7537 \mathrm{x}_{44}$

Subject to

$$
\begin{aligned}
& \mathrm{x}_{11}+\mathrm{x}_{12}+\mathrm{x}_{13}+\mathrm{x}_{14}=1, \mathrm{x}_{11}+\mathrm{x}_{21}+\mathrm{x}_{31}+\mathrm{x}_{41}=1, \\
& \mathrm{x}_{21}+\mathrm{x}_{22}+\mathrm{x}_{23}+\mathrm{x}_{24}=1, \mathrm{x}_{12}+\mathrm{x}_{22}+\mathrm{x}_{32}+\mathrm{x}_{42}=1, \\
& \mathrm{x}_{31}+\mathrm{x}_{32}+\mathrm{x}_{33}+\mathrm{x}_{34}=1, \mathrm{x}_{13}+\mathrm{x}_{22}+\mathrm{x}_{33}+\mathrm{x}_{43}=1, \\
& \mathrm{x}_{41}+\mathrm{x}_{42}+\mathrm{x}_{43}+\mathrm{x}_{44}=1, \mathrm{x}_{14}+\mathrm{x}_{24}+\mathrm{x}_{34}+\mathrm{x}_{44}=1,
\end{aligned}
$$

where $\mathrm{x}_{\mathrm{ij}} \in\{0,1\}$.

Solving it with LINGO version 6, we get the following optimal solution $\mathrm{x}_{12}{ }_{12}=\mathrm{x}_{21}{ }_{21}=\mathrm{x}_{34}{ }_{34}=\mathrm{x}_{43}{ }_{43}=1$ and others variables are zeros. Similarly for different values of $\mathrm{w}_{\mathrm{i}}$ and $\mathrm{k}_{\mathrm{i}}$, where $\mathrm{i}=1,2,3$, the obtained solutions are given in Table 5.

Table 3. A three objective Assignment problem (case of military affairs)

\begin{tabular}{|c|c|c|c|c|}
\hline C. T, Q & Task-A & Task-B & Task-C & Task-D \\
\hline \multirow{2}{*}{ FP-1 } & $(4,6,7,9)$ & $(3,5,7,10)$ & $(6,7,10,12)$ & $(3,4,6,9)$ \\
& $(7,9,11,13)$ & $(6,9,10,12)$ & $(9,10,11,12)$ & $(8,11,13,15)$ \\
& $(0.15,0.16,0.19,0.21)$ & $(0.10,0.11,0.13,0.14)$ & $(0.14,0.16,0.18,0.20)$ & $(0.05,0.07,0.09,0.11)$ \\
\hline \multirow{2}{*}{ FP-2 } & $(2,3,5,7)$ & $(5,7,8,11)$ & $(5,6,7,10)$ & $(4,7,9,11)$ \\
& $(6,7,10,12)$ & $(9,12,14,17)$ & $(7,8,10,11)$ & $(6,8,12,13)$ \\
& $(0.09,0.12,0.15,0.18)$ & $(0.14,0.16,0.18,0.20)$ & $(0.20,0.21,0.23,0.25)$ & $(0.15,0.18,0.22,0.25)$ \\
\hline \multirow{2}{*}{ FP-3 } & $(6,8,10,12)$ & $(5,7,12,14)$ & $(6,7,9,10)$ & $(4,5,7,9)$ \\
& $(3,4,5,7)$ & $(4,5,7,9)$ & $(6,7,8,11)$ & $(3,4,6,7)$ \\
& $(0.18,0.20,0.22,0.24)$ & $(0.13,0.15,0.17,0.19)$ & $(0.20,0.22,0.24,0.27)$ & $(0.15,0.16,0.18,0.20)$ \\
\hline \multirow{2}{*}{ FP-4 } & $(3,7,10,12)$ & $(6,7,10,12)$ & $(7,10,11,13)$ & $(5,7,10,14)$ \\
& $(4,6,8,10)$ & $(5,7,8,10)$ & $(4,5,7,8)$ & $(5,9,11,15)$ \\
& $(0.15,0.18,0.20,0.22)$ & $(0.19,0.21,0.23,0.25)$ & $(0.12,0.13,0.14,0.15)$ & $(0.10,0.14,0.16,0.18)$ \\
\hline
\end{tabular}


Table 4. The normalized operation cost, operation time, and ineffectiveness

\begin{tabular}{|c|c|c|c|c|}
\hline $\mathrm{FP}_{\mathrm{FP}} \mathrm{C}, \mathrm{T}, \mathrm{Q}$ & Task-A & Task-B & Task-C & Task-D \\
\hline Fighter plane-1 & $\begin{array}{c}\mathrm{k}_{1}(4,6,7,9) \\
\mathrm{k}_{2}(7,9,11,13) \\
\mathrm{k}_{3}(.15, .16, .19, .21)\end{array}$ & $\begin{array}{c}\mathrm{k}_{1}(3,5,7,10) \\
\mathrm{k}_{2}(6,9,10,12) \\
\mathrm{k}_{3}(.10, .11, .13, .14)\end{array}$ & $\begin{array}{c}\mathrm{k}_{1}(6,7,10,12) \\
\mathrm{k}_{2}(9,10,11,12) \\
\mathrm{k}_{3}(.14, .16, .18, .20)\end{array}$ & $\begin{array}{c}\mathrm{k}_{1}(3,4,6,9) \\
\mathrm{k}_{2}(8,11,13,15) \\
\mathrm{k}_{3}(.05, .07, .09, .11)\end{array}$ \\
\hline Fighter plane-2 & $\begin{array}{c}\mathrm{k}_{1}(2,3,5,7) \\
\mathrm{k}_{2}(6,7,10,12) \\
\mathrm{k}_{3}(.09, .12, .15, .18)\end{array}$ & $\begin{array}{c}\mathrm{k}_{1}(5,7,8,11) \\
\mathrm{k}_{2}(9,12,14,17) \\
\mathrm{k}_{3}(.14, .16, .18, .20)\end{array}$ & $\begin{array}{c}\mathrm{k}_{1}(5,6,7,10) \\
\mathrm{k}_{2}(7,8,10,11) \\
\mathrm{k}_{3}(.20, .21, .23, .25)\end{array}$ & $\begin{array}{c}\mathrm{k}_{1}(4,7,9,11) \\
\mathrm{k}_{2}(6,8,12,13) \\
\mathrm{k}_{3}(.15, .18, .22, .25)\end{array}$ \\
\hline Fighter plane-3 & $\begin{array}{c}\mathrm{k}_{1}(6,8,10,12) \\
\mathrm{k}_{2}(3,4,5,7) \\
\mathrm{k}_{3}(.18, .20, .22, .24)\end{array}$ & $\begin{array}{c}\mathrm{k}_{1}(5,7,12,14) \\
\mathrm{k}_{2}(4,5,7,9) \\
\mathrm{k}_{3}(.13, .15, .17, .19)\end{array}$ & $\begin{array}{c}\mathrm{k}_{1}(6,7,9,10) \\
\mathrm{k}_{2}(6,7,8,11) \\
\mathrm{k}_{3}(.20, .22, .24, .27)\end{array}$ & $\begin{array}{c}\mathrm{k}_{1}(4,5,7,9) \\
\mathrm{k}_{2}(3,4,6,7) \\
\mathrm{k}_{3}(.15, .16, .18, .20)\end{array}$ \\
\hline Fighter plane-4 & $\begin{array}{c}\mathrm{k}_{1}(3,7,10,12) \\
\mathrm{k}_{2}(4,6,8,10) \\
\mathrm{k}_{3}(.15, .18, .20, .22)\end{array}$ & $\begin{array}{c}\mathrm{k}_{1}(6,7,10,12) \\
\mathrm{k}_{2}(5,7,8,10) \\
\mathrm{k}_{3}(.19, .21, .23, .25)\end{array}$ & $\begin{array}{c}\mathrm{k}_{1}(7,10,11,13) \\
\mathrm{k}_{2}(4,5,7,8) \\
\mathrm{k}_{3}(.12, .13, .14, .15)\end{array}$ & $\begin{array}{c}\mathrm{k}_{1}(5,7,10,14) \\
\mathrm{k}_{2}(5,9,11,15) \\
\mathrm{k}_{3}(.10, .14, .16, .18)\end{array}$ \\
\hline
\end{tabular}

Table 5. Solution of FMOAP for different weight

\begin{tabular}{|c|c|c|c|c|c|}
\hline $\begin{array}{l}\text { Weight } \\
\left(\alpha_{i}\right)\end{array}$ & $\begin{array}{c}\text { Maximum value of Yager } \\
\text { Ranking Indices }\end{array}$ & $\begin{array}{c}\text { Reciprocal of } \\
\left.\text { Maximum R.I ( } \mathrm{k}_{\mathrm{i}}\right)\end{array}$ & $\begin{array}{l}\text { Multiplication } \\
\text { of weight and } \\
\operatorname{MRI}\left(\alpha_{\mathrm{i}}^{*} \mathrm{k}_{\mathrm{i}}\right)\end{array}$ & Solution & $\begin{array}{l}\text { Optimal } \\
\text { Assignment }\end{array}$ \\
\hline $\begin{array}{l}\mathrm{w}_{1}=0.2 \\
\mathrm{w}_{2}=0.3 \\
\mathrm{w}_{3}=0.5\end{array}$ & $\begin{array}{c}Y_{\mathrm{c}}^{*}(7,10,11,13)=10.75 \\
Y_{t}(9,10,11,12)=11.75 \\
Y_{\text {in }}(0.20,0.22,0.24,0.27) \\
=0.2325\end{array}$ & $\begin{array}{c}\mathrm{k}_{1}=1 / 10.75=0.0930 \\
\mathrm{k}_{2}=1 / 11.75=0.0851 \\
\mathrm{k}_{3}=1 / 0.2325=0.0432\end{array}$ & $\begin{array}{l}0.018605 \\
0.025532 \\
0.021506\end{array}$ & $\begin{array}{c}x^{*}{ }_{12}=x^{*}{ }_{21}=x^{*}{ }_{34}= \\
x_{43}^{*}=1 \text { others are } \\
\text { zero. }\end{array}$ & $\begin{array}{l}1 \rightarrow \mathrm{B}, 2 \rightarrow \mathrm{A}, \\
3 \rightarrow \mathrm{D}, 4 \rightarrow \mathrm{C} .\end{array}$ \\
\hline $\begin{array}{l}\mathrm{w}_{1}=0.1 \\
\mathrm{w}_{2}=0.2 \\
\mathrm{w}_{3}=0.7\end{array}$ & Same & $\begin{array}{c}\mathrm{k}_{1}=1 / 10.75=0.0930 \\
\mathrm{k}_{2}=1 / 11.75=0.0851 \\
\mathrm{k}_{3}=1 / 0.2325=0.0432\end{array}$ & $\begin{array}{l}0.009300 \\
0.017020 \\
0.030240\end{array}$ & $\begin{array}{c}x^{*}{ }_{14}=x^{*}{ }_{21}=x_{32}^{*}= \\
x_{43}^{*}=1 \text { and others } \\
\text { equal to zero }\end{array}$ & $\begin{array}{l}1 \rightarrow \mathrm{D}, 2 \rightarrow \mathrm{A}, \\
3 \rightarrow \mathrm{B}, 4 \rightarrow \mathrm{C}\end{array}$ \\
\hline $\begin{array}{l}\mathrm{w}_{1}=0.1 \\
\mathrm{w}_{2}=0.1 \\
\mathrm{w}_{3}=0.8\end{array}$ & Same & $\begin{array}{c}\mathrm{k}_{1}=1 / 10.75=0.0930 \\
\mathrm{k}_{2}=1 / 11.75=0.0851 \\
\mathrm{k}_{3}=1 / 0.2325=0.0432\end{array}$ & $\begin{array}{l}0.009300 \\
0.008510 \\
0.034560\end{array}$ & $\begin{array}{c}\mathrm{x}_{14}^{*}=\mathrm{x}_{21}^{*}=\mathrm{x}_{32}^{*}= \\
\mathrm{x}_{43}^{*}=1 \text { and others } \\
\text { equal to zero }\end{array}$ & $\begin{array}{l}1 \rightarrow \mathrm{D}, 2 \rightarrow \mathrm{A}, \\
3 \rightarrow \mathrm{B}, 4 \rightarrow \mathrm{C}\end{array}$ \\
\hline $\begin{array}{l}\mathrm{w}_{1}=1 / 3 \\
\mathrm{w}_{2}=1 / 3 \\
\mathrm{w}_{3}=1 / 3\end{array}$ & Same & $\begin{array}{c}\mathrm{k}_{1}=1 / 10.75=0.0930 \\
\mathrm{k}_{2}=1 / 11.75=0.0851 \\
\mathrm{k}_{3}=1 / 0.2325=0.0432\end{array}$ & $\begin{array}{l}0.03100 \\
0.02830 \\
0.01440\end{array}$ & $\begin{aligned} x_{12}^{*}= & x^{*}{ }_{21}=x_{34}^{*}= \\
x^{*}{ }_{43}= & 1 \text { others are } \\
& \text { zero. }\end{aligned}$ & $\begin{array}{l}1 \rightarrow \mathrm{B}, 2 \rightarrow \mathrm{A}, \\
3 \rightarrow \mathrm{D}, 4 \rightarrow \mathrm{C} .\end{array}$ \\
\hline
\end{tabular}

\section{CONCLUSIONS}

In this paper, we present a multi-objective assignment problem with fuzzy parameters. To solve FMOAP, we first transform the multi-objective assignment problem to an equivalent single objective assignment problem by Yager's ranking method. This method is very simple and fruitful. A numerical example relating with military affair is solved to demonstrate the efficiency and validity of the proposed method. We hope the proposed method may be useful for future study in real world FMOAPs. Here, it seems that the degree of rejection can be useful in modeling FMOAP, which indicates that the concept of intuitionistic fuzzy sets may be more effective to deal with uncertainty involved in the proposed method.

\section{ACKNOWLEDGMENTS}

The authors are very grateful to the anonymous referees for their comments and suggestions, which have led to improve the quality and presentation of the paper to its current standard. 


\section{REFERENCES}

[1] Hiller, S.F., Liberman, J. G. 2001 Introduction to operation Research, $7^{\text {th }}$ ed. Mcgraw Hill, Boston.

[2] Taha, A.H 1992 Operation Research: an introduction, $5^{\text {th }}$ ed., Macmillan, Basingstoke Hampshire.

[3] Murthy, P. R 2007 Operation Research, $2^{\text {nd }}$ ed., New Age International Limited, New Delhi.

[4] Swarup, K., Gupta, P. K., and Mohan, M. 2003 Operation Research, $11^{\text {th }}$ ed., Sultan Chand and Sons, New Delhi.

[5] Bao, C.P.,Tsai, C. M., and Tsai, M. 2007 A new approach to study the multi objective assignment problem. WHAMPOA- An inter disciplinary Journal 53, 123-132.

[6] Lin, J.C., Wen, P. U. 2004 A labeling algorithm for the fuzzy assignment problem. Fuzzy sets and Systems 142, 373-391.

[7] Chen, M. S. 1985 On a fuzzy assignment problem. Tamkang J 22, 407-411.

[8] Wang, X. Fuzzy optimal assignment problem. Fuzzy Math $3,101-108$.

[9] Mukherjee, S., Basu, K. 2010 Application of fuzzy ranking method for solving assignment problem with fuzzy costs. International Journal of Computational and Applied Mathematics 5(3), 359-368.

[10] Yager, R. R. 1981 A procedure for ordering fuzzy subsets of the unit interval. Information Sciences 24, 143-161.
[11] Geetha, S., Nair, K. P. K. 1993 A variation of assignment problem. European Journal of Operation Research 68, 422 426.

[12] Tsai, C. H., Wei, C. C., and Cheng, C. L. 1999 Multi objective fuzzy deployment of manpower. International Journal of the Computer, the Internet and Management 7(2).

[13] Kagade, K.L., Bajaj, V. H. 2010 Fuzzy method for solving multi objective assignment problem with interval cost. Journal of Statistics and Mathematics 1(1), 01-09.

[14] Kagade, K.L., Bajaj, V. H. 2009 Fuzzy approach with linear and some non-linear membership functions for solving multi-objective assignment problems. Advances in Computational Research 1(2), 14-17.

[15] Dey, P.K., Yadav, B. 2011 An Algorithm to Solve MultiObjective Assignment Problem Using Interactive Fuzzy Goal Programming Approach. Int. J. Contemp. Math. Sciences 6(34), 1651-1662.

[16] Pramanik, S., Roy, T. K. 2006 A fuzzy goal programming technique for solving multi objective transportation problem. Tamsui oxford Journal of management Sciences 22, 67-89.

[17] Pramanik, S., Roy, T. K. 2008 Multi objective transportation model with fuzzy parameters: priority based fuzzy goal programming approach. Journal of Transportation systems Engineering \& Information Technology 8(3).

[18] Zadeh L. A. 1965 Fuzzy sets, Information and Control, 338-353. 